\title{
OPEN Defect localization by an extended laser source on a hemisphere
}

\author{
Daniel Veira Canle ${ }^{1 \bowtie}$, Joni Mäkinen ${ }^{1}$, Richard Blomqvist ${ }^{1}$, Maria Gritsevich ${ }^{1,2,3}$, Ari Salmi ${ }^{1}$ \& \\ Edward Hæggström ${ }^{1}$
}

The primary goal of this study is to localize a defect (cavity) in a curved geometry. Curved topologies exhibit multiple resonances and the presence of hotspots for acoustic waves. Launching acoustic waves along a specific direction e.g. by means of an extended laser source reduces the complexity of the scattering problem. We performed experiments to demonstrate the use of a laser line source and verified the experimental results in FEM simulations. In both cases, we could locate and determine the size of a pit in a steel hemisphere which allowed us to visualize the defect on a 3D model of the sample. Such an approach could benefit patients by enabling contactless inspection of acetabular cups.

Acoustic wave generation and detection on curved geometries is challenging. Contacting techniques need to be adapted to the sample shape to effectively transmit and receive acoustic energy. Coupling sound to a sample may be hard e.g. when it is small or when sample access is restricted ${ }^{1}$. Furthermore, contacting transducers modify the boundary conditions of the sample under inspection and they are prone to oscillation ${ }^{2}$. As a result, both transmitted and received signals are distorted which make it hard to analyze the sample geometry. Practical difficulties arising from curvature include geometric focusing, and dispersion of acoustic waves. Acoustic waves generated on a spherical surface converge at the antipodal point. Measuring close to this focal point is desirable in order to achieve a high signal-to-noise ratio. The drawback in this approach is the presence of large amplitude gradients making the choice of exact measurement location critical to obtain repeatable results. Any recorded echo is masked by resonances since spherical structures are resonant geometries for acoustic waves ${ }^{3}$. Another unique feature of closed geometries is the presence of whispering gallery waves also known as circumferential waves. Circumferential waves, commonly used for pipe and cylinder inspection ${ }^{4-8}$, circumnavigate the sample multiple times and are characteristic of pipes and spheres ${ }^{3,9-12}$. Compared to spheres, hemispheres add complexity to the interpretation of wave propagation since acoustic waves reflect off their equator. The aim of this study is to develop a method to visualize damage on a hemispherical shell.

Damage detection in shells is commonly done with Lamb waves. Their wave mode profile is defined with respect to the mid-plane between the two boundaries ${ }^{13-19}$. Any tapering ${ }^{20}$ or curvature of the plate ${ }^{21-24}$ modifies the symmetry plane of the particle displacement which alters the shape and cutoff frequencies of the acoustic waves. Such modified Lamb waves are sometimes called quasi Lamb waves ${ }^{21}$. Here we generate and detect Lamb waves by laser ultrasound in a contactless manner. Laser ultrasonic methods can efficiently generate and detect acoustic waves in a rapid manner with high bandwidth and spatial resolution ${ }^{2}$. Specific sample regions can be targeted by launching directed acoustic waves with extended laser sources ${ }^{22,25,26}$. This practice is commonplace for inspection of pipes $5,8,27,28$ and Lamb waves are commonly used to characterize spherical shells ${ }^{29}$. Here we show an approach that benefits from a directed acoustic source to localize damage on a hemispherical shell.

Previously we showed that an extended line source combined with interferometric detection can locate holes as small as $(\sim 0.7 \lambda)$ on steel shells ${ }^{18}$. Here, we demonstrate how the acoustic field interacts with the cavity and we use this information to visualize the hole in a 3D model of the sample. This approach removes the need for complex scanning techniques ${ }^{30}$ and data interpretation based on mode conversion ${ }^{31}$. Such a simple approach could assist in the inspection of acetabular cups by characterizing their adhesion as well as the integrity of their attachment points ${ }^{32-34}$.

\section{Experiments}

We developed the damage detection method by studying four stainless steel hemispherical shells $(\varnothing=50 \mathrm{~mm}$ and $0.6 \mathrm{~mm}$ thickness). These structures featured holes with diameters of $9.5 \mathrm{~mm}, 4 \mathrm{~mm}$, and $2 \mathrm{~mm}$ at a polar angle $\theta=45^{\circ}$ whereas the last shell was pristine and served as a reference. We glued the samples to a $3 \mathrm{D}$ printed

${ }^{1}$ Department of Physics, Division of Material Physics, Faculty of Science, University of Helsinki, P.O.B. 64, 00014 Helsinki, Finland. 'Finnish Geospatial Research Institute, Geodeetinrinne 2, 02430 Masala, Finland. ${ }^{3}$ Institute of Physics and Technology, Ural Federal University, Mira Str. 19, 620002 Ekaterinburg, Russia. ${ }^{\square}$ email: daniel.veiracanle@helsinki.fi 

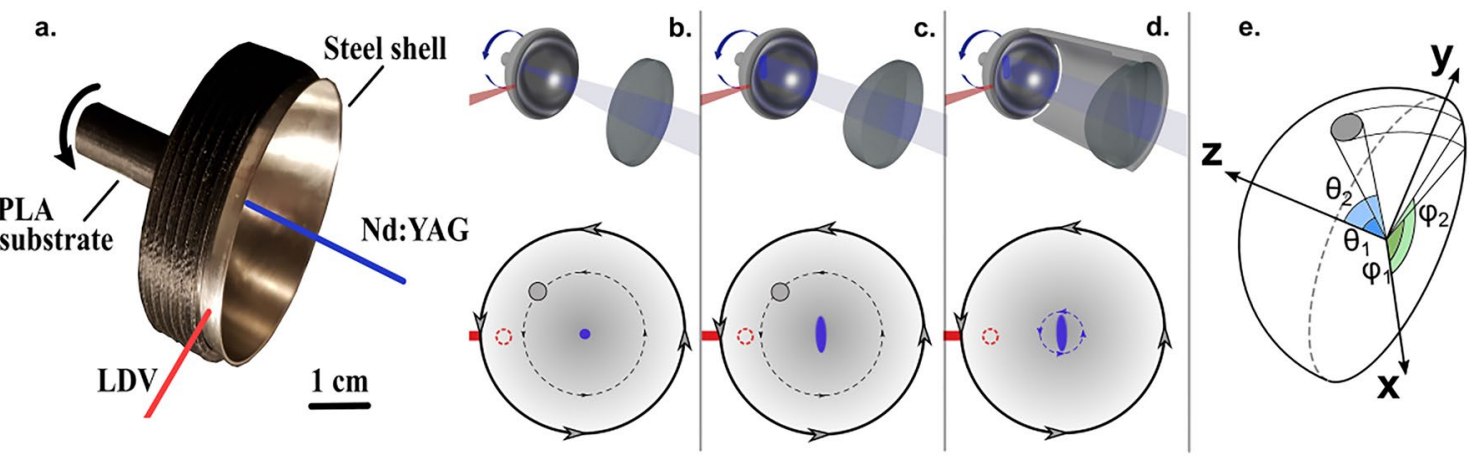

Figure 1. Experimental setup for laser ultrasound measurements. Nd:YAG laser (blue) generates guided waves on the sample whilst the laser Doppler vibrometer (LDV, red) detects them from the edge (a). The top view (bottom half) illustrates how the sample and the defect (grey circle) rotate in relation to the LDV (dashed red circle) and the Nd:YAG excitation laser (blue). A spherical (cylindrical) lens generates an acoustic point (line) source at the apex of the sample, (b-d). For localization (e), an effective approach is to use a stationary line source (c). We compare the acoustic directivity of the point and line sources on the hemispherical shell in the supplementary material of this study.
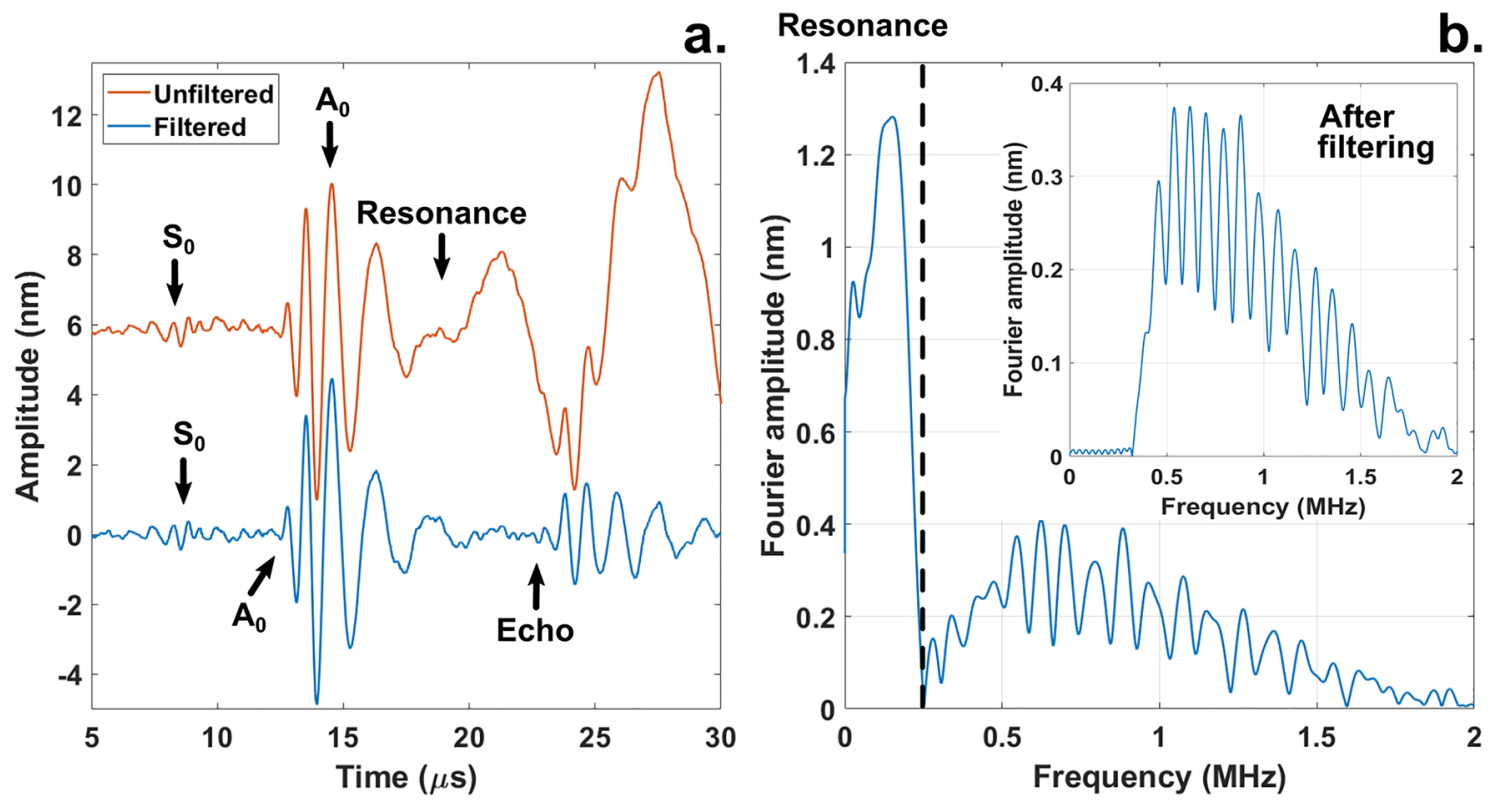

Figure 2. Filtering to remove standing waves in the structure. Signal shape produced by a point excitation on a damaged sample (a) and its frequency content (b). The raw signal (a, red, offset $6 \mathrm{~nm}$ for clarity) features a resonance that masks the echo. A high pass Fourier filter ranging from 0.1 to $0.5 \mathrm{MHz}$ (b) removes the standing wave interference and reveals an echo (a, blue).

polylactic acid (PLA) holder with epoxy (Araldite ${ }^{\circledast}$ extra strong). The entire outer surface of the shell was attached to the holder except for a $3 \mathrm{~mm}$ wide brim (Fig. 1).

The hemispherical shell completed three rotations (200 points per revolution, $1.8^{\circ}$ per step) under nonablative pulsed illumination by a Q-switched Nd:YAG laser (CFR Big Sky Laser Series, $8 \mathrm{~ns}, 40 \mathrm{~mJ}$ per pulse, $3 \mathrm{~Hz}$ pulse repetition frequency, 12 pulses per step) whilst a Polytec OFV303 (OFV3001 controller) laser Doppler Vibrometer ( $2 \mathrm{MHz}$ bandwidth in displacement mode) detected the acoustic waves at $0.8 \mathrm{~mm}$ distance from the edge of the sample. This approach generates acoustic maps by stacking signals of consecutive measurements next to each other. MATLAB [MathWorks ${ }^{\circledR}$ R2018b] generated the acoustic maps by averaging 12 signals at every point across the three rotations. To remove resonances which mask the acoustic waves, we filtered the data using a Butterworth high-pass filter (sampling frequency $62.47 \mathrm{MHz}$ for experiments and $100 \mathrm{MHz}$ for simulations, stop frequency $=0.1 \mathrm{MHz}$, pass frequency $=0.5 \mathrm{MHz}$ ). This approach reveals echoes carrying information about scatterers present in the structure (Fig. 2).

To localize damage on a $3 \mathrm{D}$ model of the hemisphere, we calculated the group velocity of the $\mathrm{A}_{0}$ mode. An algorithm extracted the time-of-arrival of the $\mathrm{A}_{0}$ mode from the data shown in Fig. 5b,e by finding the first peak of the guided wave front (Fig. 5c,f). For every azimuth we calculated the acoustic power and determined the $-3 \mathrm{~dB}$ points (Fig. 5). These points delimit the boundaries of the pit of azimuthal coordinates $\varphi_{1}$ and $\varphi_{2}$ (Fig. 1e). 


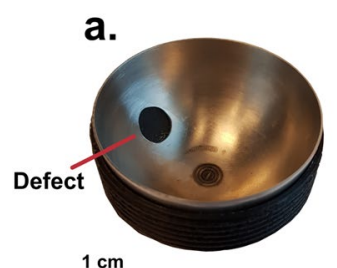

b.

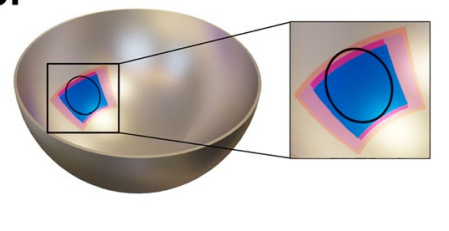

Figure 3. Comparison of a sample featuring a cavity (a) and the 3D model showing the localized defect (b). The blue and red regions indicate the size and location of a $9.5 \mathrm{~mm}$ defect predicted by the FEM simulations and the experimental results respectively. The translucent blue and red regions highlight the confidence limits of the defect size obtained from the simulations and the experiments respectively. The small mismatch in the polar direction might have been caused by a small offset produced when drilling the cavity.

We calculated the polar coordinates $\theta_{1}$ and $\theta_{2}$ from the propagation time of the echoes reflected from the defect at $\varphi=102.8^{\circ}$ and $\varphi=295.2^{\circ}$ (Fig. 5c,f). The calculation of the width and length of the defect when knowing the polar and azimuthal coordinates is straightforward and it is described in the calculations section of the supplementary material (Supplementary Fig. S2).

\section{Simulations}

We recently did finite element method (FEM) simulations (COMSOL Multiphysics ${ }^{\oplus}$ v. 5.3a) of laser-generated ultrasound on a hemispherical shell ${ }^{35}$. We studied guided waves generated by a point source, their propagation on an intact sample, and their interaction with a circular defect. In the present study we expand our simulations to include the line excitation. Videos of the FEM simulations are provided in the supplementary material to this work.

The simulation models were built the same way as in $^{35}$ for a 3D structure. COMSOL's Heat Transfer Module was coupled to the Solid Mechanics Module through thermal stresses in the time domain to generate propagating elastic waves. Both the point and line excitations were modelled as Boundary Heat Sources with profiles corresponding to the ones used in the measurements. The boundary sources were defined as two perpendicular Gaussian profiles. The line source had FWHMs of $0.90 \mathrm{~mm}$ (line width) and $7.8 \mathrm{~mm}$ (line length), and the point source FWHM was $1 \mathrm{~mm}$.

We determined the normal displacements at the surface of the sample at $1.85 \mathrm{~mm}$ distance from its edge and we assumed a point-like detection.

\section{Results}

To localize the cavity on a 3D model of a hemisphere (Fig. 3), we first launched and detected the acoustic waves from two points on the equator of the sample (Fig. 4). We chose this configuration due to ease of access, however this approach did provide insufficient information for calculating the polar angles of the defect. Instead, we placed the detection point on the equator and the excitation location at the apex of the hemisphere. This approach yielded acoustic maps (Fig. 5) showing echoes radiating from the scatterer which allowed us to estimate the defect size and location (Table 1).

\section{Discussion and conclusions}

The defect localization (Fig. 3) is made possible by the extended laser source. Such an acoustic source generates a planar wave package whose shadowing by a defect allows one to calculate the width of the region containing the scatterer. The width of the smallest detectable defect is determined by the size of the line source and the size of the scanning steps. In practice, this technique is applicable down to defect sizes equal to half the width of the acoustic source $(3.9 \mathrm{~mm})$. This has been verified both numerically and experimentally by studying defects of sizes down to $2 \mathrm{~mm}^{18}$ (Table 1, Supplementary Fig. S3). Each scanning step corresponded to an azimuthal rotation of $1.8^{\circ}$ or $0.6 \mathrm{~mm}$ at the defect location. The calculation of the length of the defect can be frustrated by the long tails of the antisymmetric Lamb waves mode (Fig. 2). In our case, the tail from the direct wave would cover the echo radiating from the defect if the distance from the detection point to the scatterer would be smaller than $3 \mathrm{~mm}$. Beyond these limitations another fundamental limiting factor is the wavelength of the interferometer. The OFV303 operated in displacement mode ( $2 \mathrm{MHz}$ bandwidth) and considering the group velocity of the antisymmetric mode (Supplementary Eq. S2), the detectability limit is roughly $1.5 \mathrm{~mm}$.

A challenge of using laser interferometry for damage localization is laser alignment. To achieve a high signalto-noise ratio (SNR), the laser beam from the LDV must be perpendicular to the surface of the sample. The shortest optimum distance for the OFV303 is $232 \mathrm{~mm}$. The leverage caused by this distance means that a 11.4' tilt in the laser beam translates as a $1 \mathrm{~mm}$ distance on the surface of the sample. This is the reason why the distance from the LDV to the brim of the hemisphere is different for simulations and experiments. We performed multiple experiments to properly align the LDV and obtain a high SNR but decided not to repeat the simulations since they are computationally heavy taking about a month of computer time in total.

In this study, the radius of the hemisphere is much larger than the central acoustic wavelength $\lambda \sim 3 \mathrm{~mm}$. Therefore, the impact of curvature on wave modes is negligible and we treat these vibrations as Lamb waves. The uniqueness of the problem arises when the excitation and the detection locations lie on the equator at diametrically opposite locations (Fig. 4). This region comprises a convergence point for acoustic waves, a point that 
a.

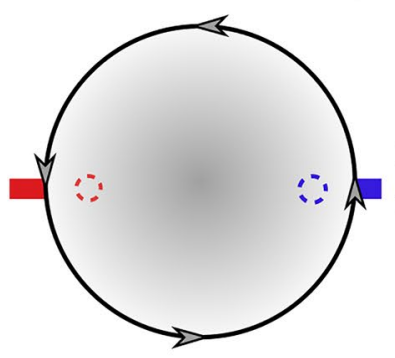

e.

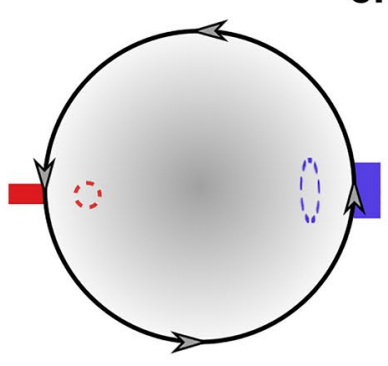

b.
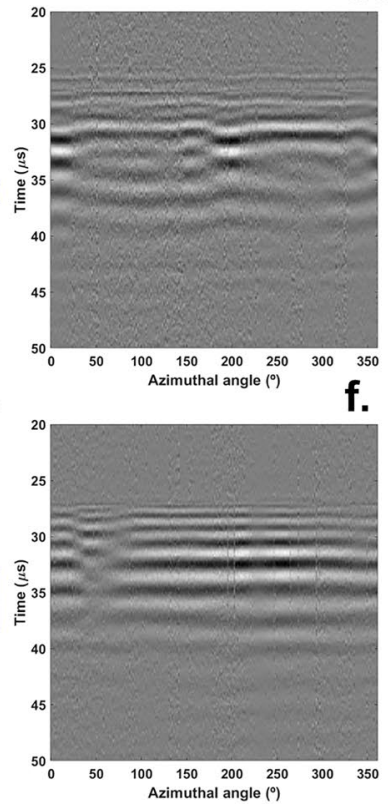

\begin{abstract}
(1)
\end{abstract}

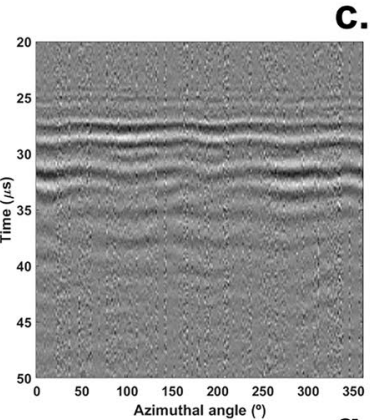

Azimuthal angle (o) $\mathbf{g}$.

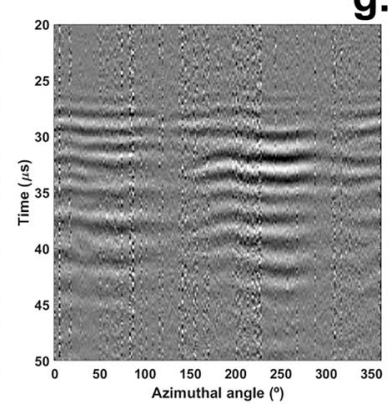

C.
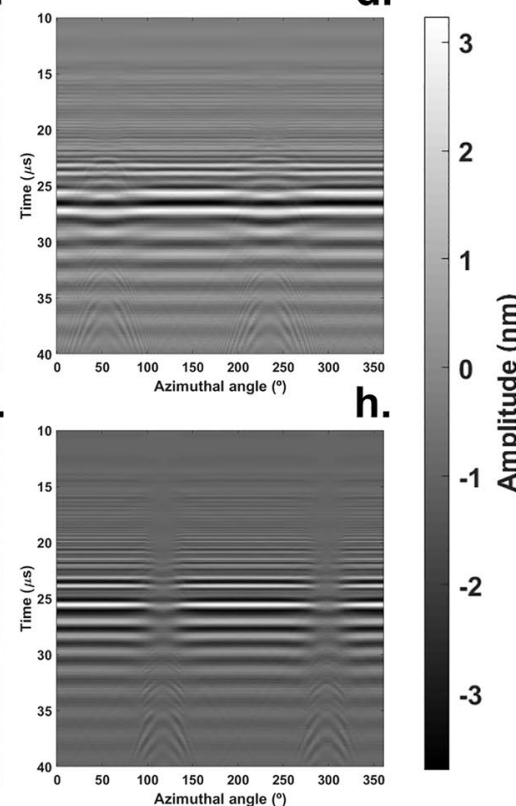

3

2

1

焉

0

Figure 4. Detection of Lamb waves originated at the equator of the hemisphere. The Nd:YAG laser (dashed blue ellipse) generated acoustic waves from the edge of the hemisphere and the LDV aimed at the opposite end (dashed red circle) measured the vibrations (a,e). The acoustic maps consist of 200 signals stacked next to each other where the $\mathrm{x}$-axis represents the azimuthal angle, the $\mathrm{y}$-axis the propagation time of the acoustic waves and the color gradient the wave amplitude. Upper row: Lamb waves generated with a point laser source; Bottom row: Lamb waves generated with a laser line source. Intact sample (b,f); damaged sample with a $9.5 \mathrm{~mm}$ pit, experimental results $(\mathbf{c}, \mathbf{g})$ and FEM simulations $(\mathbf{d}, \mathbf{h})$. Data have been filtered to remove resonances. The laser line source produces a planar wave front whose shadowing $(\mathbf{g}, \mathbf{h})$ highlights the presence of a defect. In this configuration, there are insufficient data for calculating the polar angles since the echoes emanating from the defect are not visible in the experimental context $(\mathbf{g})$.

features large gradients in the acoustic field amplitude (Supplementary Video 1). Consequentially, even small deviations in detection position make the experiment challenging to reproduce. Furthermore, our FEM simulations show that due to the reflections from the equator at these wavelengths, there is a region where the surface displacement is a node yielding low signal-to-noise ratio (Supplementary Fig. S2). We alleviated these issues by selecting the apex of the hemisphere as the source of acoustic waves while keeping the detection location on the brim of the hemisphere (Fig. 5).

Inspection of spheres and hemispheres suffer from interference caused by infinite paths of the same length connecting the poles on the shell surface. Path selection by directed acoustic fields minimizes the multipath interference. We further reduced this problem by moving the excitation location to the apex of the dome since, in the case of hemispheres, multipath interference only emerges when the detection and the excitation locations lie on the equator (Fig. 4).

As explained by Shui ${ }^{3}$, spheres are inherently resonant geometries for acoustic waves because of geometric focusing. When acoustic attenuation is low, acoustic waves converge at the antipodal point and circulate the surface of the hemisphere multiple times (Supplementary Video 2). Knowing the group velocity of the A0 mode, the resonance frequency is approximately $f_{\text {res }}=\frac{c_{A 0}}{R}$ where $c_{A_{0}}$ and $\mathrm{R}$ are the group velocity of the A0 mode and $\mathrm{R}$ the radius of the hemisphere. In our case $f_{\text {res }}=120 \mathrm{kHz}$ which is close to the observed value $f_{\text {res }}=162 \mathrm{kHz}$. One could incorrectly interpret resonant modes as propagating waves leading to wrong conclusions. Filtering the data reveals an echo hiding under the resonance (Fig. 2). Knowing the time-of-flight of the echo and its angular width allowed us to estimate the size of the defect as well as its location (Table 1).

Despite the limitations of the introduced technique, the proposed approach extracts enough information to localize a defect in a 3D model of the sample (Fig. 3). Our method localized damage in curved geometries while minimizing interference caused by curvature. The contactless nature of the technique preserves the information of the propagating wave front since the sample boundaries are not loaded by contacting transducers ${ }^{2}$. Launching acoustic waves with an extended line source allowed us to localize a defect without the need of mapping the acoustic field across the surface of the sample ${ }^{36}$. The inspection of curved structures with buried defects could benefit from these advantages since it could be possible to image defects despite access being restricted ${ }^{29,32}$. 
a.
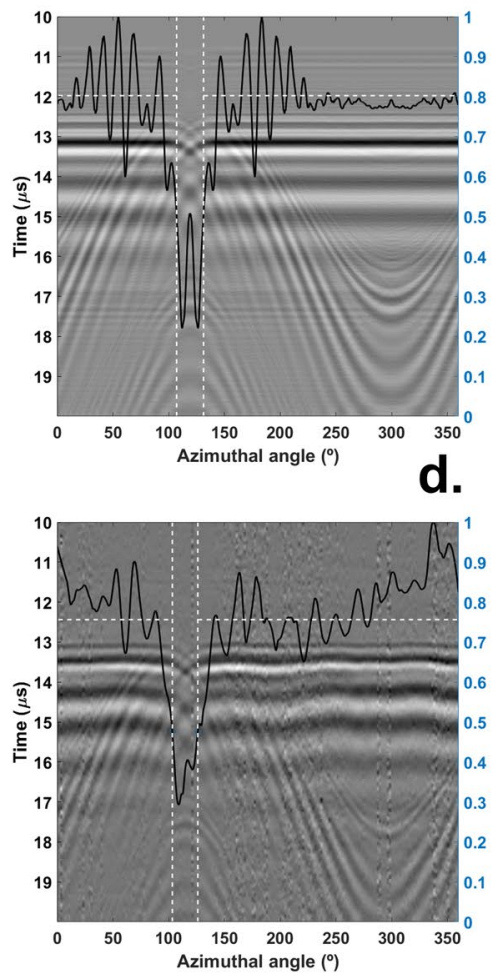

b.
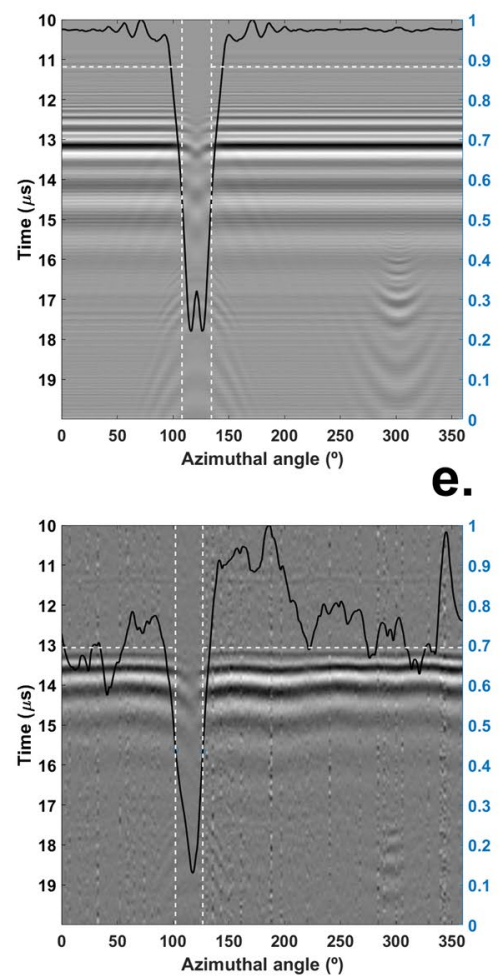

c.

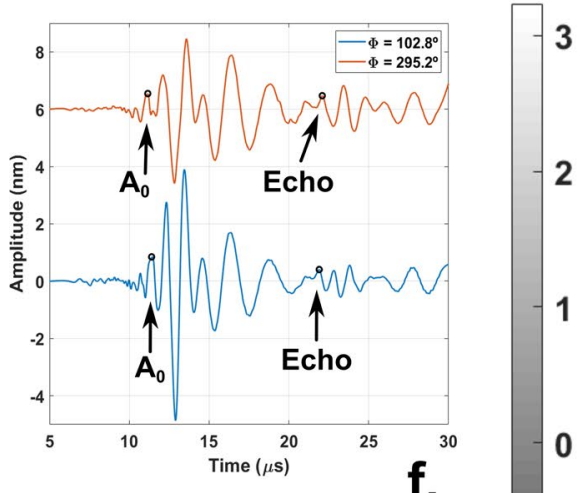

2

1

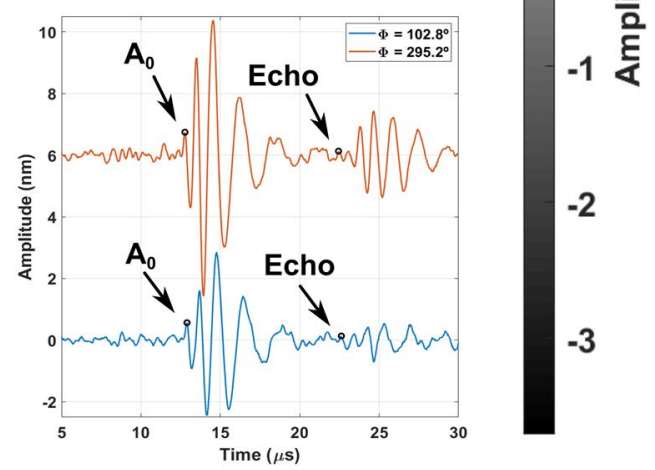

Figure 5. Detection of Lamb waves originated from the apex of the hemisphere. The acoustic maps (a,b,d,e) consist of 200 signals stacked next to each other where the $x$-axis represents the azimuthal angle, the $y$-axis the propagation time of the acoustic waves and the color gradient the wave amplitude. First row: Experimental results; second row: simulation results (a,d point source, b,e line source). c,f: echoes arising from a $9.5 \mathrm{~mm}$ defect on a laser line source scan at $\varphi=102.8^{\circ}$ and its antipodal location $\varphi=295.2^{\circ}$. The black lines in $(\mathbf{b}, \mathbf{e})$ represent the acoustic power at different azimuths. The horizontal white lines represent its mean value across the undamaged region while the vertical lines are the $-3 \mathrm{~dB}$ points. Data have been filtered to remove standing waves.

\begin{tabular}{|c|c|c|c|c|c|}
\hline \multicolumn{2}{|c|}{$9.5 \mathrm{~mm}$ defect } & \multicolumn{2}{|c|}{$4 \mathrm{~mm}$ defect } & \multicolumn{2}{|c|}{$2 \mathrm{~mm}$ defect } \\
\hline \multicolumn{6}{|c|}{ Polar angles $\left({ }^{\circ}\right)$} \\
\hline $55.74 \pm 0.23$ & $34.26 \pm 0.23$ & $49.58 \pm 0.23$ & $40.42 \pm 0.23$ & $47.29 \pm 0.23$ & $42.71 \pm 0.23$ \\
\hline $56.2 \pm 1.9$ & $34.0 \pm 1.9$ & $52.2 \pm 3.7$ & $37.8 \pm 3.7$ & $49.4 \pm 3.4$ & $40.6 \pm 3.4$ \\
\hline $55.2 \pm 2.0$ & $35.8 \pm 2.0$ & $50.2 \pm 3.7$ & $39.8 \pm 3.7$ & $49.0 \pm 3.7$ & $41.0 \pm 3.7$ \\
\hline \multicolumn{6}{|c|}{ Azimuths $\left({ }^{\circ}\right)$} \\
\hline $105.99 \pm 0.16$ & $136.42 \pm 0.16$ & $114.72 \pm 0.16$ & $127.68 \pm 0.16$ & $117.96 \pm 0.16$ & $124.44 \pm 10.16$ \\
\hline $102.0 \pm 6.5$ & $127.7 \pm 6.5$ & $115.9 \pm 2.7$ & $126.5 \pm 2.7$ & - & - \\
\hline $108.0 \pm 5.0$ & $134.4 \pm 5.0$ & $114.0 \pm 2.7$ & $128.4 \pm 2.7$ & $110.2 \pm 4.2$ & $132.2 \pm 4.2$ \\
\hline Length, I (mm) & Width, w (mm) & Length, I (mm) & Width, w (mm) & Length, I (mm) & Width, w (mm) \\
\hline $9.39 \pm 0.10$ & $9.39 \pm 0.10$ & $4.00 \pm 0.10$ & $4.00 \pm 0.10$ & $2.00 \pm 0.10$ & $2.00 \pm 0.10$ \\
\hline $9.7 \pm 1.4$ & $7.9 \pm 2.8$ & $6.3 \pm 2.3$ & $3.3 \pm 1.2$ & $3.8 \pm 2.1$ & - \\
\hline $8.5 \pm 1.4$ & $8.2 \pm 2.2$ & $4.2 \pm 2.3$ & $4.4 \pm 1.2$ & $3.5 \pm 2.3$ & $6.8 \pm 1.8$ \\
\hline
\end{tabular}

Table 1. Defect location and size in the true location, simulated, and experimental cases for the $9.5 \mathrm{~mm}, 4 \mathrm{~mm}$ and $2 \mathrm{~mm}$ holes. From top to bottom, expected (green), experimental (orange), and simulation results (blue). Length and width are the dimensions of the sector containing the defect along both, the azimuthal and polar directions (Fig. 1e). The azimuthal coordinates as well as the width for the $2 \mathrm{~mm}$ defect in the experimental case are missing since there was no visible shadowing of the acoustic waveforms by the cavity (Supplementary Fig. S3). The magnitudes feature confidence limits of one standard deviation. The corresponding uncertainty calculations are presented in the supplementary material. 
Received: 25 April 2021; Accepted: 5 July 2021

Published online: 26 July 2021

\section{References}

1. Canle, D. V., Salmi, A. \& Haeggstrom, E. Flaw detection in rotating structures by Lamb waves. in 2016 IEEE International Ultrasonics Symposium (IUS) 1-3 (IEEE, 2016). doi:https://doi.org/10.1109/ULTSYM.2016.7728645

2. C.B Scruby \& L.E Drain. Laser ultrasonics, techniques and applications. NDT \& E International 24, (2003).

3. Shui, Y., Royer, D., Dieulesaint, E. \& Sun, Z. Resonance of surface waves on spheres. in Ultrasonics Symposium Proceedings 1, 343-346 (Publ by IEEE, 1988).

4. Leonard, K. R. \& Hinders, M. K. Lamb wave tomography of pipe-like structures. Ultrasonics 43, 574-583 (2005).

5. Gao, W., Glorieux, C. \& Thoen, J. Study of circumferential waves and their interaction with defects on cylindrical shells using line-source laser ultrasonics. J. Appl. Phys. 91, 6114 (2002).

6. Valle, C., Niethammer, M., Qu, J. \& Jacobs, L. J. Crack characterization using guided circumferential waves. J. Acoust. Soc. Am. 110, 1282-1290 (2001).

7. Clorennec, D. \& Royer, D. Analysis of surface acoustic wave propagation on a cylinder using laser ultrasonics. Appl. Phys. Lett. 82, 4608-4610 (2003).

8. Lee, J.-H. \& Lee, S.-J. Application of laser-generated guided wave for evaluation of corrosion in carbon steel pipe. NDT E Int. 42, 222-227 (2009).

9. Abid, A., Pereira, D., Fernandes, J. C. \& Belanger, P. Sensitivity study of ultrasonic guided waves to cortical bone mechanical properties with axial and circumferential propagation. Acta Acust. united with Acust. 103, 421-429 (2017).

10. Li, Z., He, C., Liu, Z. \& Wu, B. Quantitative detection of lamination defect in thin-walled metallic pipe by using circumferential Lamb waves based on wavenumber analysis method. NDT E Int. https://doi.org/10.1016/j.ndteint.2018.11.005 (2019).

11. Park, J., Lee, J., Jeong, S. G. \& Cho, Y. A study on guided wave propagation in a long distance curved pipe. J. Mech. Sci. Technol. 33, 4111-4117 (2019).

12. Clorennec, D. \& Royer, D. Investigation of surface acoustic wave propagation on a sphere using laser ultrasonics. Appl. Phys. Lett. 85, 2435-2437 (2004).

13. Chang, Z. \& Mal, A. Scattering of Lamb waves from a rivet hole with edge cracks. Mech. Mater. 31, 197-204 (1999).

14. McKeon, J. C. P. \& Hinders, M. K. Lamb wave scattering from a through hole. J. Sound Vib. 224, 843-862 (1999).

15. Grahn, T. Lamb wave scattering from a circular partly through-thickness hole in a plate. Wave Motion 37, 63-80 (2003).

16. Yeasin Bhuiyan, M., Shen, Y. \& Giurgiutiu, V. Interaction of Lamb waves with rivet hole cracks from multiple directions. Proc. Inst. Mech. Eng. Part C J. Mech. Eng. Sci. 231, 2974-2987 (2017).

17. Diligent, O. \& Lowe, M. J. S. Reflection of the s0 Lamb mode from a flat bottom circular hole. J. Acoust. Soc. Am. 118, 2869-2879 (2005).

18. Veira Canle, D., Makinen, J., Gritsevich, M., Salmi, A. \& Haeggstrom, E. Localization of millimeter size defects in a steel hemispherical shell. in IEEE International Ultrasonics Symposium, IUS 2019-Octob, 1575-1578 (IEEE Computer Society, 2019).

19. Viktorov, I. A. Rayleigh and Lamb Waves Physical Theory and Applications (Springer, 2014).

20. Nurmalia, N., Ogi, H., Hirao, M. \& Nakahata, K. Mode conversion behavior of SH guided wave in a tapered plate. NDT E Int. 45, 156-161 (2012)

21. Fong, K. L. J. A Study of Curvature Effects on Guided Elastic Waves (Imperial College, 2005).

22. Hutchins, D. A., Dewhurst, R. J. \& Palmer, S. B. Directivity patterns of laser-generated ultrasound in aluminum. Cit. J. Acoust. Soc. Am. 70, 1362 (1981)

23. Royer, D., Dieulesaint, E., Jia, X. \& Shui, Y. Optical generation and detection of surface acoustic waves on a sphere. Appl. Phys. Lett. 52, 706-708 (1988).

24. Liu, G. \& Qu, J. Guided circumferential waves in a circular annulus. J. Appl. Mech. 65, 424 (1998).

25. Aindow, A. M., Dewhurst, R. J. \& Palmer, S. B. Laser-generation of directional surface acoustic wave pulses in metals. Opt. Commun. 42, 116-120 (1982).

26. Kim, H., Jhang, K., Shin, M. \& Kim, J. A noncontact NDE method using a laser generated focused-Lamb wave with enhanced defect-detection ability and spatial resolution. NDT E Int. 39, 312-319 (2006).

27. Liu, X. K., Yang, S. X. \& Liu, Y. Q. Numerical Study for Surface-breaking Crack Detection on a Cylinder Using Laser-generated Ultrasound. in 2018 15th International Conference on Ubiquitous Robots, UR 2018 797-802 (Institute of Electrical and Electronics Engineers Inc., 2018). https://doi.org/10.1109/URAI.2018.8441815

28. Cès, M., Royer, D. \& Prada, C. Characterization of mechanical properties of a hollow cylinder with zero group velocity Lamb modes. J. Acoust. Soc. Am. 132, 180-185 (2012).

29. Sugino, C., Ruzzene, M. \& Erturk, A. Experimental and computational investigation of guided waves in a human skull. Ultrasound Med. Biol. 47, 787-798 (2021).

30. McKie, A. D. W. \& Addison, R. C. Laser-based ultrasonic inspection of complexly contoured rocket engine components. Review of Progress in Quantitative Nondestructive Evaluation https://doi.org/10.1007/978-1-4615-5339-7_77 (2012).

31. Veira Canle, D., Salmi, A. \& Hæggström, E. Non-contact damage detection on a rotating blade by Lamb wave analysis. NDT E Int. 92, 159-166 (2017).

32. Ahmed, G. A. et al. How screw connections influence the primary stability of acetabular cups under consideration of different bone models. J. Orthop. 21, 302-308 (2020).

33. Alshuhri, A. A., Holsgrove, T. P., Miles, A. W. \& Cunningham, J. L. Development of a non-invasive diagnostic technique for acetabular component loosening in total hip replacements. Med. Eng. Phys. 37, 739-745 (2015).

34. Alshuhri, A. A., Holsgrove, T. P., Miles, A. W. \& Cunningham, J. L. Non-invasive vibrometry-based diagnostic detection of acetabular cup loosening in total hip replacement (THR). Med. Eng. Phys. 48, 188-195 (2017).

35. Mäkinen, J., Veira Canle, D., Blomqvist, R., Salmi, A. \& Haeggström, E. Simulation of Laser-excited Surface Acoustic Waves Traveling on A Hemispherical Steel Shell. in (COMSOL, 2018).

36. Selim, H. et al. Defect reconstruction by non-destructive testing with laser induced ultrasonic detection. Ultrasonics 101, 106000 (2020).

\section{Author contributions}

D.V.C. planned the experiments together with A.S. and J.M. R.B. prepared the samples and together with DVC executed the experiments. D.V.C. analyzed the experimental data which was supported by J.M.s FEM simulations. D.V.C. and M.G. developed the equations that allowed localizing and sizing the defect. D.V.C. wrote the first draft of the manuscript and J.M., M.G., A.S. and E.H. contributed to finalizing it for publication.

\section{Funding}

Open access funded by University of Helsinki Library. 


\section{Competing interests}

The authors declare no competing interests.

\section{Additional information}

Supplementary Information The online version contains supplementary material available at https://doi.org/ 10.1038/s41598-021-94084-w.

Correspondence and requests for materials should be addressed to D.V.C.

Reprints and permissions information is available at www.nature.com/reprints.

Publisher's note Springer Nature remains neutral with regard to jurisdictional claims in published maps and institutional affiliations.

(c) (1) Open Access This article is licensed under a Creative Commons Attribution 4.0 International License, which permits use, sharing, adaptation, distribution and reproduction in any medium or format, as long as you give appropriate credit to the original author(s) and the source, provide a link to the Creative Commons licence, and indicate if changes were made. The images or other third party material in this article are included in the article's Creative Commons licence, unless indicated otherwise in a credit line to the material. If material is not included in the article's Creative Commons licence and your intended use is not permitted by statutory regulation or exceeds the permitted use, you will need to obtain permission directly from the copyright holder. To view a copy of this licence, visit http://creativecommons.org/licenses/by/4.0/.

(C) The Author(s) 2021 